\title{
Necessary and sufficient quantum information characterization of Einstein-Podolsky-Rosen steering
}

\author{
Marco Piani ${ }^{1,2}$ and John Watrous ${ }^{3}$ \\ ${ }^{1}$ Institute for Quantum Computing $\&$ Department of Physics and Astronomy, \\ University of Waterloo, 200 University Avenue West, Waterloo, Ontario N2L 3G1, Canada \\ ${ }^{2}$ Department of Physics and SUPA, University of Strathclyde, Glasgow G4 ONG, UK \\ ${ }^{3}$ Institute for Quantum Computing 8 School of Computer Science, University of Waterloo, \\ 200 University Avenue West, Waterloo, Ontario N2L 3G1, Canada
}

\begin{abstract}
Steering is the entanglement-based quantum effect that embodies the "spooky action at a distance" disliked by Einstein and scrutinized by Einstein, Podolsky, and Rosen. Here we provide a necessary and sufficient characterization of steering, based on a quantum information processing task: the discrimination of branches in a quantum evolution, which we dub subchannel discrimination. We prove that, for any bipartite steerable state, there are instances of the quantum subchannel discrimination problem for which this state allows a correct discrimination with strictly higher probability than in absence of entanglement, even when measurements are restricted to local measurements aided by one-way communication. On the other hand, unsteerable states are useless in such conditions, even when entangled. We also prove that the above steering advantage can be exactly quantified in terms of the steering robustness, which is a natural measure of the steerability exhibited by the state.
\end{abstract}

PACS numbers: 03.67.Mn, 03.67.Bg, 03.65.Ud

Entanglement is a property of distributed quantum systems that does not have a classical counterpart and challenges our everyday-life intuition about the physical world [1]. It also is the key element in many quantum information processing tasks [2]. The strongest feature exhibited by entangled systems is non-locality [3]. A weaker feature related to entanglement is steering: roughly speaking, in quantum steering one party can induce very different ensembles for the local state of the other party, beyond what is possible based only on a conceivable classical knowledge about the other party's "hidden state" $[4,5]$. Steering embodies the "spooky action at a distance" - in the words of Einstein [6] - identified by Schroedinger [7], scrutinized by Einstein, Podolsky, and Rosen [8], and formally put on sound ground in $[4,5]$. Not all entangled states are steerable, and not all steerable states exhibit nonlocality $[4,5]$, but states that exhibit steering allow for the verification of their entanglement in a semi-device independent way: there is no need to trust the devices used by the steering party $[4,5,9]$. Besides its foundational interest, steering is interesting in practice in bipartite tasks, like quantum key distribution (QKD) [10], where it is convenient or appropriate to trust the devices of one of two parties, but not necessarily of the other one. For example, by exploiting steering, key rates unachievable in a fully device-independent approach [11] are possible, still assuming less about the devices than in a standard QKD approach [12]. For these reasons, steering has recently attracted significant interest, both theoretically and experimentally [13-30], mostly directed to the verification of steering. Nonetheless, an answer to the question "What is steering useful for?" can arguably be considered limited $[9,12]$. Further- more, the quantification of steering has just started to be addressed [24].

In this Letter we fully characterize and quantify steering in an operational way that mirrors the asymmetric features of steering, and that breaks new ground in the investigation of the usefulness of steering. We prove that every steerable state is a resource in a quantum information task that we dub subchannel discrimination, in a practically relevant scenario where measurements can only be performed locally. Subchannel discrimination is the identification of which branch of a quantum evolution a quantum system undergoes (see Fig. 1). It is well known that entanglement between a probe and an ancilla can help in discriminating different channels [31-44]. In [45] it was proven that every entangled state is useful in some instance of the subchannel discrimination problem. Ref. [46] analyzed the question of whether such an advantage is preserved when joint measurements on the output probe and the ancilla are not possible. Here we prove that, when only local measurements coordinated by forward classical communication are possible, every steerable state remains useful, while non-steerable entangled states become useless. We further prove that this usefulness, optimized over all instances of the subchannel discrimination problem, is exactly equal to the robustness of steering, a natural way of quantifying steering using techniques similar to the ones used in [24], but based on the notion of robustness [47-50].

Preliminaries: entanglement and steering. - We will denote by a ^ (hat) mathematical entities that are "normalized." So, for example, a positive semidefinite operator with unit trace is a (normalized) state $\hat{\rho}$. An ensemble $\mathcal{E}=\left\{\rho_{a}\right\}_{a}$ for a state $\hat{\rho}$ is a collection of substates $\rho_{a} \leq \hat{\rho}$ 
such that $\sum_{a} \rho_{a}=\hat{\rho}$. Each substate $\rho_{a}$ is proportional to a normalized state $\hat{\rho}_{a}, \rho_{a}=p_{a} \hat{\rho}_{a}$, with $p_{a}=\operatorname{Tr}\left(\rho_{a}\right)$ the probability of $\hat{\rho}_{a}$ in the ensemble. An assemblage $\mathcal{A}=\left\{\mathcal{E}_{x}\right\}_{x}=\left\{\rho_{a \mid x}\right\}_{a, x}$ is a collection of ensembles $\mathcal{E}_{x}$ for the same state $\hat{\rho}$, one for each $x$, i.e., $\sum_{a} \rho_{a \mid x}=\hat{\rho}$, for all $x$. For example, $\mathcal{E}=\left\{\frac{1}{2}|0\rangle\left\langle 0\left|, \frac{1}{2}\right| 1\right\rangle\langle 1|\right\}$ and $\mathcal{E}^{\prime}=$ $\left\{\frac{1}{2}|+\rangle\left\langle+\left|, \frac{1}{2}\right|-\right\rangle\langle-|\right\}$, with $| \pm\rangle:=(|0\rangle \pm|1\rangle) / \sqrt{2}$, are both ensembles for the maximally mixed state $\mathbb{1} / 2$ of a qubit, and taken together they form an assemblage $\mathcal{A}=\left\{\mathcal{E}, \mathcal{E}^{\prime}\right\}$ for $\mathbb{1} / 2$. Along similar lines, a measurement assemblage $\mathcal{M A}=\left\{M_{a \mid x}\right\}_{a, x}$ is a collection of positive operators $M_{a \mid x} \geq 0$ satisfying $\sum_{a} M_{a \mid x}=\mathbb{1}$ for each $x$, which thus represents one positive-operator-valued measure (or POVM), describing a quantum measurement, for each $x$. For a fixed bipartite state $\hat{\rho}_{A B}$, every measurement assemblage on Alice leads to an assemblage on Bob via

$$
\rho_{a \mid x}^{B}=\operatorname{Tr}_{A}\left(M_{a \mid x}^{A} \hat{\rho}_{A B}\right) .
$$

On the other hand, every assemblage on Bob $\left\{\sigma_{a \mid x}\right\}_{a, x}$ has a quantum realization (1) for some $\hat{\rho}_{A B}$ satisfying $\hat{\rho}_{B}=\operatorname{Tr}_{A}\left(\hat{\rho}_{A B}\right)=\sum_{x} \sigma_{a \mid x}=: \hat{\sigma}_{B}$ and for some measurement assemblage [51].

An assemblage $\mathcal{A}=\left\{\rho_{a \mid x}\right\}_{a, x}$ is unsteerable if

$$
\rho_{a \mid x}^{\mathrm{US}}=\sum_{\lambda} p(\lambda) p(a \mid x, \lambda) \hat{\sigma}(\lambda)=\sum_{\lambda} p(a \mid x, \lambda) \sigma(\lambda),
$$

for all $a, x$, for some probability distribution $p(\lambda)$, conditional probability distributions $p(a \mid x, \lambda)$, and states $\hat{\sigma}(\lambda)$. Here $\lambda$ indicates a (hidden) classical random variable, and we introduced also subnormalized states $\sigma(\lambda)=$ $p(\lambda) \hat{\sigma}(\lambda)$. We observe that every conditional probability distribution $p(a \mid x, \lambda)$ can be written as a convex combination of deterministic conditional probability distributions: $p(a \mid x, \lambda)=\sum_{\nu} p(\nu \mid \lambda) D(a \mid x, \nu)$, where $D(a \mid x, \nu)=$ $\delta_{a, f_{\nu}(x)}$ is a deterministic response function labeled by $\nu$. This means that, by a suitable relabeling,

$$
\rho_{a \mid x}^{\mathrm{US}}=\sum_{\lambda} D(a \mid x, \lambda) \sigma(\lambda) \quad \forall a, x,
$$

where the summation is over labels of deterministic response functions. We say that an assemblage $\left\{\rho_{a \mid x}\right\}_{a, x}$ is steerable if it is not unsteerable. A separable (or unentangled) state decomposes as $\hat{\sigma}_{A B}^{\text {sep }}=$ $\sum_{\lambda} p(\lambda) \hat{\sigma}_{A}(\lambda) \otimes \hat{\sigma}_{B}(\lambda)$, for $\hat{\sigma}_{A}(\lambda), \hat{\sigma}_{B}(\lambda)$ local states, $\lambda$ a classical label, and $p(\lambda)$ a probability distribution [52]. A state is entangled if it is not separable. An unsteerable assemblage can always be obtained via (1) from the separable state $\rho_{A B}=\sum_{\lambda} p(\lambda)|\lambda\rangle\left\langle\left.\lambda\right|_{A} \otimes \hat{\sigma}(\lambda)_{B}\right.$, with $M_{a \mid x}=\sum_{\mu} p(a \mid x, \mu)|\mu\rangle\langle\mu|$, and $\langle\mu \mid \lambda\rangle=\delta_{\mu \lambda}$. Most importantly, any separable state can only lead to unsteerable assemblages, as, for a separable state, one has $\operatorname{Tr}_{A}\left(M_{a \mid x} \sigma_{A B}^{\mathrm{sep}}\right)=\sum_{\lambda} p(\lambda) p(a \mid x, \lambda) \sigma_{B}(\lambda)$, with $p(a \mid x, \lambda)=\operatorname{Tr}_{A}\left(M_{a \mid x} \sigma_{A}(\lambda)\right)$. It follows that entanglement is a necessary condition for steerability, and, in

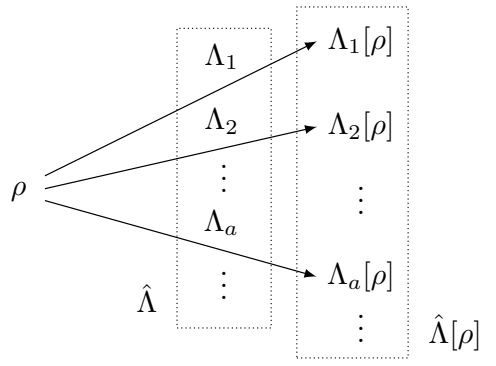

FIG. 1: A decomposition of a channel into subchannels can be seen as a decomposition of a quantum evolution into branches of the evolution. If $\left\{\Lambda_{a}\right\}_{a}$ is an instrument for $\hat{\Lambda}$, then we can imagine that the evolution $\rho \mapsto \hat{\Lambda}[\rho]$ has branches $\rho \mapsto \Lambda_{a}[\rho]$, where each branch takes place with probability $\operatorname{Tr}\left(\Lambda_{a}[\rho]\right)$. The transformation described by the total channel $\hat{\Lambda}$ can be seen as the situation where the "which-branch" information is lost. An example of subchannel discrimination problem is that of distinguishing between the two quantum evolutions $\Lambda_{i}[\rho]=$ $K_{i} \rho K_{i}^{\dagger}, i=0,1$, with $K_{0}=|0\rangle\langle 0|+\sqrt{1-\gamma}| 1\rangle\langle 1|$ and $K_{1}=$ $\sqrt{\gamma}|0\rangle\langle 1|$, corresponding to the so-called amplitude damping channel $\hat{\Lambda}=\Lambda_{0}+\Lambda_{1}[2]$.

turn, a steerable assemblage is a clear signature of entanglement. Interestingly, not all entangled states lead to steerable assemblages by the action of appropriate local measurement assemblages $[4,5]$; we call steerable states those that do, and unsteerable states those that do not. There exist entangled states that are steerable by one party but not the other (see, e.g., [22]). In this Letter, when we refer to a state being steerable or unsteerable, it is always to be assumed that Alice is the steering party.

Channel and subchannel identification. - A subchannel $\Lambda$ is a linear completely positive map that is trace nonincreasing: $\operatorname{Tr}(\Lambda[\rho]) \leq \operatorname{Tr}(\rho)$, for all states $\rho$. If a subchannel $\Lambda$ is trace-preserving, $\operatorname{Tr}(\Lambda[\rho])=\operatorname{Tr}(\rho)$, for all $\rho$, we use the ${ }^{\wedge}$ notation and say that $\hat{\Lambda}$ is a channel. An instrument $\mathcal{I}=\left\{\Lambda_{a}\right\}_{a}$ for a channel $\hat{\Lambda}$ is a collection of subchannels $\Lambda_{a}$ such that $\hat{\Lambda}=\sum_{a} \Lambda_{a}$ (see Figure 1). Every instrument has a physical realization, where the index $a$ can be considered available to some party $[2,53,54]$.

Fix an instrument $\left\{\Lambda_{a}\right\}_{a}$ for a channel $\Lambda$, and consider a measurement $\left\{Q_{b}\right\}_{b}$ on the output space of $\hat{\Lambda}$. The joint probability of $\Lambda_{a}$ and $Q_{b}$ for input $\rho$ is $p(a, b):=$ $\operatorname{Tr}\left(Q_{b} \Lambda_{a}[\rho]\right)=p(b \mid a) p(a)$, where $p(a)=\operatorname{Tr}\left(\Lambda_{a}[\rho]\right)$ is the probability of the subchannel $\Lambda_{a}$ for the given input $\rho$ and $p(b \mid a)=p(a, b) / p(a)$ is the conditional probability of the outcome $b$ given that the subchannel $\Lambda_{a}$ took place (see Figure 2(a)). The probability of correctly identifying which subchannel was realized is

$$
p_{\text {corr }}\left(\left\{\Lambda_{a}\right\}_{a},\left\{Q_{b}\right\}_{b}, \rho\right)=\sum_{a} \operatorname{Tr}\left(Q_{a} \Lambda_{a}[\rho]\right) .
$$

The archetypal case of subchannel discrimination is that of channel discrimination, where $\Lambda_{a}=p_{a} \hat{\Lambda}_{a}$, with channels $\hat{\Lambda}_{a}$ and probabilities $p_{a}$. The problem often considered is that of telling apart just two channels 


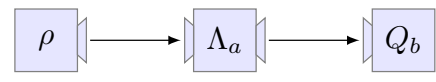

(a)

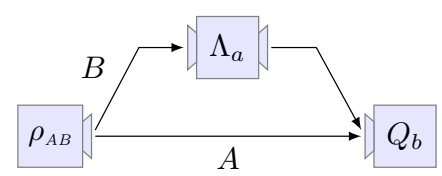

(b)

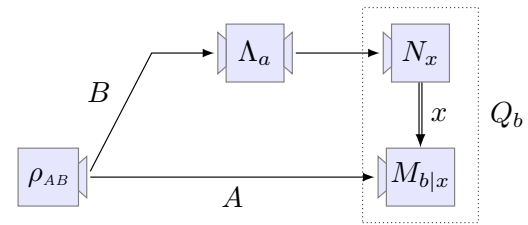

(c)

FIG. 2: Different strategies for subchannel discrimination. (a) No entanglement is used: a probe, initially in the state $\rho$, undergoes the quantum evolution $\hat{\Lambda}$, with branches $\Lambda_{a}$, and is later measured, with an outcome $b$ for the measurement described by the POVM $\left\{Q_{b}\right\}_{b}$, which is the guess for which branch of the evolution actually took place. (b) The probe $B$ is potentially entangled with an ancilla $A$; the output probe and the ancilla are jointly measured. (c) The probe is still potentially entangled with an ancilla, but the final measurement $\left\{Q_{b}\right\}_{b}$ is restricted to local measurements on the output probe and the ancilla, coordinated by one-way classical communication (single lines represent quantum systems, double lines classical information): the outcome $x$ of the measurement $\left\{N_{x}\right\}_{x}$ performed on the output probe is used to decide which measurement $\left\{M_{b \mid x}\right\}_{b}$ to perform on the ancilla.

$\hat{\Lambda}_{0}$ and $\hat{\Lambda}_{1}$, each given with probability $p_{0}=p_{1}=$ $1 / 2$. In this case the total (average) channel is simply $\hat{\Lambda}=\frac{1}{2} \hat{\Lambda}_{0}+\frac{1}{2} \hat{\Lambda}_{1}$. The best success probability in identifying subchannels $\left\{\Lambda_{a}\right\}_{a}$ with an input $\rho$ is defined as $p_{\text {corr }}\left(\left\{\Lambda_{a}\right\}_{a}, \rho\right):=\max _{\left\{Q_{b}\right\}_{b}} p_{\text {corr }}\left(\left\{\Lambda_{a}\right\}_{a},\left\{Q_{b}\right\}_{b}, \rho\right)$. Optimizing also over the input state, one arrives at $p_{\text {corr }}^{\mathrm{NE}}\left(\left\{\Lambda_{a}\right\}_{a}\right):=\max _{\rho} p_{\text {corr }}\left(\left\{\Lambda_{a}\right\}_{a}, \rho\right)$, where the superscript NE stands for "no entanglement" (see Fig. 2(a)).

Indeed, one may try to improve the success probability by using an entangled input state $\rho_{A B}$ of an input probe $B$ and an ancilla $A$. The guess about which subchannel took place is based on a joint measurement of the output probe and the ancilla (see Fig. 2(b)), with success probability $p_{\text {corr }}\left(\left\{\Lambda_{a}^{B}\right\}_{a},\left\{Q_{b}^{A B}\right\}_{b}, \rho_{A B}\right)$. In the latter expression we have explicitly indicated that the subchannels act non-trivially only on $B$, while input state and measurement pertain to $A B$. One can define the optimal probability of success for a scheme that uses input entanglement and global measurements: $p_{\text {corr }}^{\mathrm{E}}\left(\left\{\Lambda_{a}\right\}_{a}\right):=$ $\max _{\rho_{A B}} \max _{\left\{Q_{b}^{A B}\right\}_{b}} p_{\text {corr }}\left(\left\{\Lambda_{a}^{B}\right\}_{a},\left\{Q_{b}^{A B}\right\}_{b}, \rho_{A B}\right)$. We say that entanglement is useful in discriminating subchannels $\left\{\Lambda_{a}\right\}_{a}$ if $p_{\text {corr }}^{\mathrm{E}}\left(\left\{\Lambda_{a}\right\}_{a}\right)>p_{\text {corr }}^{\mathrm{NE}}\left(\left\{\Lambda_{a}\right\}_{a}\right)$. It is known that there are instances of subchannel discrimination, already in the simple setting $\left\{\Lambda_{a}\right\}_{a}=\left\{\frac{1}{2} \hat{\Lambda}_{0}, \frac{1}{2} \hat{\Lambda}_{0}\right\}$, where $p_{\text {corr }}^{\mathrm{E}} \approx 1 \gg p_{\text {corr }}^{\mathrm{NE}} \approx 0$ (see [46] and references therein).

In [45] it was proven that, for any entangled state $\rho_{A B}$, there exists a choice $\left\{\frac{1}{2} \hat{\Lambda}_{0}, \frac{1}{2} \hat{\Lambda}_{1}\right\}$ such that

$$
p_{\text {corr }}\left(\left\{\frac{1}{2} \hat{\Lambda}_{0}, \frac{1}{2} \hat{\Lambda}_{1}\right\}, \rho_{A B}\right)>p_{\text {corr }}^{\mathrm{NE}}\left(\left\{\frac{1}{2} \hat{\Lambda}_{0}, \frac{1}{2} \hat{\Lambda}_{1}\right\}\right),
$$

i.e., that every entangled state is useful for the task of (sub)channel discrimination. In this sense, every entangled state, independently of how weakly entangled it is, is a resource. Nonetheless, exploiting such a resource may require arbitrary joint measurements on the output probe and ancilla [46]. From a conceptual perspective, one may want to limit measurements to those performed by local operations and classical communication (LOCC), as this makes the input entangled state the only non-local resource. This limitation can be justified also from a practical perspective: LOCC measurements are arguably easier to implement, and might be the only feasible kind of measurements, especially in a scenario where only weakly entangled states can be produced. We do not know whether every entangled state stays useful for subchannel discrimination when measurements are restricted to be LOCC, but we will see that, if the measurements are limited to local operations and forward communication (one-way LOCC), then only steerable states remain useful.

Steerability and subchannel identification by means of restricted measurements. - A Bob-to-Alice one-way LOCC measurement $\mathcal{M}^{B \rightarrow A}=\left\{Q_{a}^{B \rightarrow A}\right\}_{a}$ has the structure $Q_{a}^{B \rightarrow A}=\sum_{x} M_{a \mid x}^{A} \otimes N_{x}^{B}$, where $\left\{N_{x}^{B}\right\}_{x}$ is a measurement on $B$ and $\left\{M_{a \mid x}^{A}\right\}_{a, x}$ is a measurement assemblage on $A$. We define $p_{\text {corr }}^{B \rightarrow A}\left(\mathcal{I}, \rho_{A B}\right):=$ $\max _{\mathcal{M}^{B \rightarrow A}} p_{\text {corr }}\left(\mathcal{I}^{B}, \mathcal{M}^{B \rightarrow A}, \rho_{A B}\right)$ as the optimal probability of success in the discrimination of the instrument $\mathcal{I}^{B}=\left\{\Lambda_{a}^{B}\right\}_{a}$ by means of the input state $\rho_{A B}$ and oneway LOCC measurements from $B$ to $A$ (see Fig. 2(c)). We say that $\rho_{A B}$ is useful in this restricted-measurement scenario if $p_{\text {corr }}^{B \rightarrow A}\left(\mathcal{I}, \rho_{A B}\right)>p_{\text {corr }}^{\mathrm{NE}}(\mathcal{I})$ for some instrument $\mathcal{I}$ [59]. Using (1), we find that

$$
p_{\text {corr }}\left(\mathcal{I}^{B}, \mathcal{M}^{B \rightarrow A}, \rho_{A B}\right)=\sum_{a, x} \operatorname{Tr}_{B}\left(\Lambda_{a}^{\dagger B}\left[N_{x}^{B}\right] \rho_{a \mid x}\right),
$$

where $\Lambda_{a}^{\dagger}$ denotes the dual map to $\Lambda_{a}$, defined via $\operatorname{Tr}\left(X \Lambda_{a}[Y]\right)=\operatorname{Tr}\left(\Lambda_{a}^{\dagger}[X] Y\right), \forall X, Y$ (assuming $\Lambda_{a}$ is completely positive). If the assemblage $\mathcal{A}=\left\{\rho_{a \mid x}\right\}_{a, x}$ appearing in (5) is unsteerable, then we can achieve an equal or better performance with an uncorrelated probe in the best input state $\hat{\sigma}(\lambda)$ among the ones appearing in Eq. (2). Thus, if $\rho_{A B}$ is unsteerable, then it is useless for subchannel discrimination with one-way measurements. This applies also to entangled states that are unsteerable, which are nonetheless useful in channel discrimination with arbitrary measurements [45].

We will now prove that every steerable state is useful in subchannel discrimination with one-way-LOCC measurements. To state our result in full detail we need to 
introduce the steering robustness of $\rho_{A B}$,

$$
R_{\mathrm{steer}}^{A \rightarrow B}\left(\rho_{A B}\right):=\sup _{\mathcal{M} \mathcal{A}} R(\mathcal{A}),
$$

where the supremum is over all measurement assemblages $\mathcal{M A}=\left\{M_{a \mid x}\right\}_{a, x}$ on $A, R(\mathcal{A})$ is the steering robustness of the assemblage $\mathcal{A}$,

$$
\begin{array}{r}
R(\mathcal{A}):=\min \left\{t \geq 0 \mid\left\{\frac{\rho_{a \mid x}+t \tau_{a \mid x}}{1+t}\right\}_{a, x}\right. \text { unsteerable, } \\
\left.\left\{\tau_{a \mid x}\right\} \text { an assemblage }\right\},
\end{array}
$$

and $\mathcal{A}$ is obtained from $\rho_{A B}$ with the measurement assemblage $\mathcal{M A}$ on $A$ (see Eq. (1)). The steering robustness of $\mathcal{A}$ is a measure of the minimal "noise" needed to destroy the steerability of the assemblage $\mathcal{A}$, with noise intended as the mixing with an arbitrary assemblage $\left\{\tau_{a \mid x}\right\}_{a, x}$. We prove the following.

Theorem 1. Every steerable state is useful in one-way subchannel discrimination. More precisely, it holds

$$
\sup _{\mathcal{I}} \frac{p_{\text {corr }}^{B \rightarrow A}\left(\mathcal{I}, \rho_{A B}\right)}{p_{c o r r}^{\mathrm{NE}}(\mathcal{I})}=R_{\text {steer }}^{A \rightarrow B}\left(\rho_{A B}\right)+1
$$

where the supremum is over all instruments $\mathcal{I}$.

Proof. Using the definitions (6) and (7) one checks [55]

$$
p_{\text {corr }}\left(\mathcal{I}^{B}, \mathcal{M}^{B \rightarrow A}, \rho_{A B}\right) \leq\left(1+R_{\text {steer }}^{A \rightarrow B}\left(\rho_{A B}\right)\right) p_{\text {corr }}^{\mathrm{NE}}(\mathcal{I}),
$$

for any $\mathcal{M}^{B \rightarrow A}$ and any $\mathcal{I}$. We will prove next that the bound can be approximated arbitrarily well. We will do so by constructing appropriate instances of the subchannel discrimination problem. To do this, we will need that the steering robustness $R(\mathcal{A})$ of any assemblage $\mathcal{A}=\left\{\rho_{a \mid x}\right\}_{a, x}$ can be calculated via semidefinite programming (SDP) [56]. In particular, we prove [55] that $R(\mathcal{A})+1$ is equal to the optimal value of the SDP optimization problem

$$
\begin{aligned}
\text { maximize } & \sum_{a, x} \operatorname{Tr}\left(F_{a \mid x} \rho_{a \mid x}\right) \\
\text { subject to } & \sum_{a, x} D(a \mid x, \lambda) F_{a \mid x} \leq \mathbb{1} \quad \forall \lambda \\
& F_{a \mid x} \geq 0 \quad \forall a, x,
\end{aligned}
$$

where the $\lambda$ 's label the deterministic response functions.

Now, let $\mathcal{M A}=\left\{M_{a \mid x}\right\}_{a, x}$ be a measurement assemblage on $A$, and $\mathcal{A}$ the resulting assemblage on $B$. Let $F_{a \mid x}$ be optimal, i.e., such that $\sum_{a, x} \operatorname{Tr}\left(F_{a \mid x} \rho_{a \mid x}\right)=$ $1+R(\mathcal{A})$. Define linear maps $\Lambda_{a}$ via their duals, as

$$
\begin{aligned}
\Lambda_{a}^{\dagger} & =\Lambda_{a}^{\dagger} \circ \Pi_{X} & & \forall a, \\
\Lambda_{a}^{\dagger}[|x\rangle\langle x|] & =\alpha F_{a \mid x} & & \forall a, x .
\end{aligned}
$$

Here $\circ$ is composition, and $\Pi_{X}$ indicates the projector onto an orthonormal basis $\{|x\rangle\}, x=1, \ldots,|X|$, where $|X|$ is the number of settings in the measurement assemblage $\mathcal{M A}$. The constant $\alpha>0$ will be chosen soon. Because of the conditions (9c), (10), and (11), the $\Lambda_{a}^{\dagger}$ 's are completely positive linear maps, hence the $\Lambda_{a}$ 's are too; they act according to $\Lambda_{a}[\rho]=$ $\alpha \sum_{x} \operatorname{Tr}\left(F_{a \mid x} \rho\right)|x\rangle\langle x|$, and are subchannels as long as $\sum_{a} \Lambda_{a}^{\dagger}[\mathbb{1}]=\sum_{a, x} \Lambda_{a}^{\dagger}[|x\rangle\langle x|]=\alpha \sum_{a, x} F_{a \mid x} \leq \mathbb{1}$, a condition that can be satisfied for $\alpha=\left\|\sum_{a, x} F_{a \mid x}\right\|_{\infty}^{-1}$, with $\|\cdot\|_{\infty}$ the operator norm. We introduce $N$ additional subchannels, defined as $\Lambda_{a}[\rho]=\frac{1}{N} \operatorname{Tr}\left(\left(\mathbb{1}-\sum_{a} \Lambda_{a}^{\dagger}[\mathbb{1}]\right) \rho\right) \hat{\sigma}_{a}$, for $a=|A|+1, \ldots,|A|+N$, where $|A|$ indicates the original number of outcomes for POVMs in $\mathcal{M A}$, and $\hat{\sigma}_{a}$ are arbitrary states in a two-dimensional space orthogonal to $\operatorname{span}\{|x\rangle|x=1, \ldots| X \mid$,$\} . The subchannels$ $\Lambda_{a}, a=1, \ldots,|A|+N$ define an instrument $\mathcal{I}$ for the trace-preserving channel $\hat{\Lambda}=\sum_{a=1}^{|A|+N} \Lambda_{a}$, and one can incorporate the measurement assemblage $\mathcal{M A}$ into a oneway LOCC strategy $\mathcal{M}^{B \rightarrow A}$ such that $\alpha(1+R(\mathcal{A})) \leq$ $p_{\text {corr }}\left(\mathcal{I}^{B}, \mathcal{M}^{B \rightarrow A}, \rho_{A B}\right) \leq \alpha(1+R(\mathcal{A}))+\frac{2}{N}[55]$. On the other hand, condition (9b) implies [55] $\alpha \leq p_{\text {corr }}^{\mathrm{NE}}(\mathcal{I}) \leq$ $\alpha+\frac{2}{N}$, so $p_{\text {corr }}\left(\mathcal{I}^{B}, \mathcal{M}^{B \rightarrow A}, \rho_{A B}\right) / p_{\text {corr }}^{\mathrm{NE}}(\mathcal{I}) \geq \frac{1+R(\mathcal{A})}{1+2 /(\alpha N)}$. The claim follows from letting $N$ be arbitrarily large.

Conclusions. - We have proven that the steerable states are precisely the states useful for the task of subchannel discrimination with feed-forward local measurements. This answers a question left open by [46] about the characterization of a large class of entangled states that remain useful for (sub)channel discrimination with local measurements. Most importantly, it provides a full operational characterization - and proof of usefulness - of steering in terms of a fundamental task, subchannel discrimination, in a setting - that of restricted measurements - very relevant from the practical point of view. The construction in the proof of Theorem 1 proves that, for any measurement assemblage $\mathcal{M A}$ on $A$ such that the corresponding $\mathcal{A}$ exhibit steering with robustness $R(\mathcal{A})>0$, there exist instances of the subchannel discrimination problem with restricted measurements where the use of the steerable state ensures a probability of success approximately $(1+R(\mathcal{A}))$-fold higher than in the case where no entanglement is used. Thus, the robustnesses $R(\mathcal{A})$ and $R_{\text {steer }}^{A \rightarrow B}\left(\rho_{A B}\right)$ have operational meanings not only in terms of the resilience of steerability versus noise, but also in applicative terms. Also, they constitute semi-device-independent lower bounds on the generalized robustness of entanglement $R_{g}\left(\rho_{A B}\right)=\min \{t \geq$ $0 \mid \frac{\rho_{A B}+t \tau_{A B}}{1+t}$ separable, $\tau$ a state $\}[48,49]$ :

$$
R(\mathcal{A}) \leq R_{\text {steer }}^{A \rightarrow B}\left(\rho_{A B}\right) \leq R_{g}\left(\rho_{A B}\right) .
$$

That (12) holds is immediate, given definitions (6) and (7) and the fact that a separable state leads always to unsteerable assemblages. Notice that $R_{g}$ is an entanglement 
measure with operational interpretations itself $[57,58]$. We believe that the way to quantify steerability that we have introduced is finer-grained than the approach of [24], while preserving the computational efficiency deriving from the use of semidefinite programming. For example, while the so-called steering weight of [24] is such that all pure entangled states, however weekly entangled, are deemed maximally steerable, because of (12) we know that weakly entangled pure states have small steering robustness [49]. On the other hand, maximally entangled states $\psi_{d}^{+}$for large local dimension $d$ do have large steering robustness. Indeed, we prove [55] that, if $d$ is some power of a prime number, then $R_{\text {steer }}^{A \rightarrow B}\left(\psi_{d}^{+}\right) \geq \sqrt{d}-2$.

Many questions remain open for further investigation: a closed formula for the steerability robustness of pure (maximally entangled) states; whether the result of Theorem 1 can be strengthened to prove that every steerable state is useful for channel-rather than general subchannel-discrimination with restricted measurements; whether general LOCC (rather than one-way LOCC) measurements can restore the usefulness of all entangled states for (sub)channel discrimination.

Acknowledgments. - We acknowledge useful discussions and correspondence with D. Cavalcanti and M. Pusey. We also acknowledge support from NSERC and CIFAR.

[1] R. Horodecki, P. Horodecki, M. Horodecki, and K. Horodecki, Rev. Mod. Phys. 81, 865 (2009), URL http://link.aps.org/doi/10.1103/RevModPhys. 81.865.

[2] M. A. Nielsen and I. L. Chuang (2010).

[3] N. Brunner, D. Cavalcanti, S. Pironio, V. Scarani, and S. Wehner, Rev. Mod. Phys. 86, 419 (2014), URL http: //link.aps.org/doi/10.1103/RevModPhys.86.419.

[4] H. M. Wiseman, S. J. Jones, and A. C. Doherty, Phys. Rev. Lett. 98, 140402 (2007), URL http://link.aps. org/doi/10.1103/PhysRevLett.98.140402.

[5] S. J. Jones, H. M. Wiseman, and A. C. Doherty, Phys. Rev. A 76, 052116 (2007), URL http://link.aps.org/ doi/10.1103/PhysRevA.76.052116.

[6] M. Born, The Born-Einstein Letters (Walker, 1971).

[7] E. Schrödinger, Mathematical Proceedings of the Cambridge Philosophical Society 31, 555 (1935), ISSN 14698064, URL http://journals. cambridge.org/article_ S0305004100013554.

[8] A. Einstein, B. Podolsky, and N. Rosen, Physical review 47, 777 (1935).

[9] E. G. Cavalcanti, M. J. W. Hall, and H. M. Wiseman, Phys. Rev. A 87, 032306 (2013), URL http://link. aps. org/doi/10.1103/PhysRevA.87.032306.

[10] N. Gisin, G. Ribordy, W. Tittel, and H. Zbinden, Rev. Mod. Phys. 74, 145 (2002), URL http://link. aps .org/ doi/10.1103/RevModPhys.74.145.

[11] A. Acín, N. Brunner, N. Gisin, S. Massar, S. Pironio, and V. Scarani, Phys. Rev. Lett. 98, 230501 (2007), URL http://link.aps.org/doi/10.1103/PhysRevLett.98.
230501.

[12] C. Branciard, E. G. Cavalcanti, S. P. Walborn, V. Scarani, and H. M. Wiseman, Phys. Rev. A 85, 010301 (2012), URL http://link.aps.org/doi/10. 1103/PhysRevA.85.010301.

[13] E. G. Cavalcanti, S. J. Jones, H. M. Wiseman, and M. D. Reid, Phys. Rev. A 80, 032112 (2009), URL http:// link.aps.org/doi/10.1103/PhysRevA.80.032112.

[14] D. J. Saunders, S. J. Jones, H. M. Wiseman, and G. J. Pryde, Nat Phys 6, 845 (2010), URL http://dx.doi. org/10.1038/nphys 1766 .

[15] Z. Y. Ou, S. F. Pereira, H. J. Kimble, and K. C. Peng, Phys. Rev. Lett. 68, 3663 (1992), URL http://link. aps.org/doi/10.1103/PhysRevLett.68.3663.

[16] W. P. Bowen, R. Schnabel, P. K. Lam, and T. C. Ralph, Phys. Rev. Lett. 90, 043601 (2003), URL http://link. aps.org/doi/10.1103/PhysRevLett.90.043601.

[17] D. H. Smith, G. Gillett, M. P. de Almeida, C. Branciard, A. Fedrizzi, T. J. Weinhold, A. Lita, B. Calkins, T. Gerrits, H. M. Wiseman, et al., Nature communications 3, 625 (2012).

[18] A. J. Bennet, D. A. Evans, D. J. Saunders, C. Branciard, E. G. Cavalcanti, H. M. Wiseman, and G. J. Pryde, Phys. Rev. X 2, 031003 (2012), URL http://link.aps.org/ doi/10.1103/PhysRevX.2.031003.

[19] V. Händchen, T. Eberle, S. Steinlechner, A. Samblowski, T. Franz, R. F. Werner, and R. Schnabel, Nature Photonics 6, 596 (2012).

[20] S. Steinlechner, J. Bauchrowitz, T. Eberle, and R. Schnabel, Phys. Rev. A 87, 022104 (2013), URL http:// link.aps.org/doi/10.1103/PhysRevA.87.022104.

[21] B. Wittmann, S. Ramelow, F. Steinlechner, N. K. Langford, N. Brunner, H. M. Wiseman, R. Ursin, and A. Zeilinger, New Journal of Physics 14, 053030 (2012).

[22] J. Bowles, T. Vértesi, M. T. Quintino, and N. Brunner, Physical Review Letters 112, 200402 (2014).

[23] M. F. Pusey, Phys. Rev. A 88, 032313 (2013), URL http: //link.aps.org/doi/10.1103/PhysRevA.88.032313.

[24] P. Skrzypczyk, M. Navascués, and D. Cavalcanti, Phys. Rev. Lett. 112, 180404 (2014), URL http://link.aps. org/doi/10.1103/PhysRevLett.112.180404.

[25] S. Jevtic, M. F. Pusey, D. Jennings, and T. Rudolph, arXiv preprint arXiv:1303.4724 (2013).

[26] A. Milne, S. Jevtic, D. Jennings, H. Wiseman, and T. Rudolph, arXiv preprint arXiv:1403.0418 (2014).

[27] T. Moroder, O. Gittsovich, M. Huber, and O. Gühne, arXiv preprint arXiv:1405.0262 (2014).

[28] J. Schneeloch, C. J. Broadbent, S. P. Walborn, E. G. Cavalcanti, and J. C. Howell, Physical Review A 87, 062103 (2013).

[29] J. Schneeloch, P. B. Dixon, G. A. Howland, C. J. Broadbent, and J. C. Howell, Physical review letters 110, 130407 (2013).

[30] J. Schneeloch, C. J. Broadbent, and J. C. Howell, Physics Letters A (2014).

[31] A. Kitaev, Russ. Math. Surv. 52, 1191 (1997).

[32] V. Paulsen, Completely Bounded Maps and Operator Algebras, Cambridge Studies in Advanced Mathematics (Cambridge University Press, 2002).

[33] A. Childs, J. Preskill, and J. Renes, J. Mod. Opt. 47, 155 (2000).

[34] G. M. D'Ariano, P. LoPresti, and M. G. A. Paris, Phys. Rev. Lett. 87, 270404 (2001).

[35] A. Acin, Phys. Rev. Lett. 87, 177901 (2001). 
[36] V. Giovannetti, S. Lloyd, and L. Maccone, Science 306, 1330 (2004).

[37] A. Gilchrist, N. K. Langford, and M. A. Nielsen, Phys. Rev. A 71, 062310 (2005).

[38] B. Rosgen and J. Watrous, in Proc. 20th Ann. Conf. Comp. Compl. (2005), pp. 344-354.

[39] M. F. Sacchi, Phys. Rev. A 71, 062340 (2005).

[40] M. F. Sacchi, Phys. Rev. A 72, 014305 (2005).

[41] S. Lloyd, Science 321, 1463 (2008).

[42] S. Pirandola, Phys. Rev. Lett. 106, 090504 (2011).

[43] S. Pirandola, New J. Phys. 13, 113012 (2011).

[44] S. T. et al., Phys. Rev. Lett. 101, 253601 (2008).

[45] M. Piani and J. Watrous, Phys. Rev. Lett. 102, 250501 (pages 4) (2009).

[46] W. Matthews, M. Piani, and J. Watrous, Phys. Rev. A 82, 032302 (2010), URL http://link.aps.org/doi/10. 1103/PhysRevA.82.032302.

[47] G. Vidal and R. Tarrach, Phys.Rev. A 59, 141 (1999).

[48] M. Steiner, Phys. Rev. A 67, 054305 (2003).

[49] A. Harrow and M. Nielsen, Phys. Rev. A 68, 012308 (2003).

[50] J. Geller and M. Piani, arXiv preprint arXiv:1401.8197 (2014).

[51] L. P. Hughston, R. Jozsa, and W. K. Wootters, Physics Letters A 183, 14 (1993), ISSN 0375-
9601, URL http://www.sciencedirect.com/science/ article/pii/0375960193908809.

[52] R. F. Werner, Phys. Rev. A 40, 4277 (1989), URL http: //link.aps.org/doi/10.1103/PhysRevA.40.4277.

[53] E. B. Davies and J. T. Lewis, Communications in Mathematical Physics 17, 239 (1970).

[54] M. Horodecki, Quantum information and computation 1, 3 (2001).

[55] See Supplemental Material at [URL will be inserted by publisher] for details.

[56] S. P. Boyd and L. Vandenberghe, Convex optimization (Cambridge university press, 2004).

[57] F. G. S. L. Brandão and N. Datta, IEEE Transactions on Information Theory 57, 1754 (2011).

[58] F. G. S. L. Brandão, Phys. Rev. A 76, 030301(R) (2007).

[59] Notice that no bipartite state $\rho_{A B}$ is useful in one-way subchannel identification when the communication goes from the ancilla to the output probe. This is because the initial measurement of the ancilla simply creates an ensemble of input substates for the channel, and we might as well choose the best input to begin with. So, the only one-way communication that may have a non-trivial effect is that from the output probe to the ancilla. 